\title{
Doença de Hodgkin e AIDS
}

\author{
AIDS and Hodgkin's Disease
}

Luciana Barreto

\begin{abstract}
Resumo
Apesar de não ser doença definidora de AIDS, o risco relativo (RR) de DH é definitivamente maior em pelo menos alguns subgrupos de pacientes com infecção pelo H IV (HIVpos).

U ma vez que se evidenciou haver surgido uma entidade clínica com características gerais distintas da D H , procurou-se estabelecer o perfil epidemiológico da D H-H IV; determinar se haveria um subgrupo específico de grande risco para o desenvolvimento desta nova entidade, ou al gum subgrupo em que não ela ocorresse; definir o perfil biológico tumoral e a possibilidade dessas alterações influenciarem características clínicas, o perfil de resposta terapêutica e a sobrevida livre de doença.

C omo os pacientes $\mathrm{H}$ IV pos se apresentam com D oença de $\mathrm{H}$ odgkin mais agressiva e alterações imunológicas que comprometem a resposta terapêutica obtida na população geral, a estratégia de tratamento para eles tem mudado, de forma a atingir índices de remissão completa (RC) e sobrevida a longo prazo maiores com o uso de esquemas anti-retrovirais altamente eficazes, adaptações de esquemas quimioterápicos antigos e novos protocolos terapêuticos.

0 presente artigo apresenta e analisa os fatores que se encontram envolvidos na patogênese e terapêutica da D H -H IV.
\end{abstract}

Palavras-chave: D oença de Hodgkin; HIV; EBV; epidemiologia; patologia; terapia.

${ }^{1}$ M édica do Serviço de Hematologia do H ospital do Câncer I, Instituto Nacional de Câncer, Praça Cruz Vermelha 23, Centro; 20231-000 Rio de Janeiro, RJ - Brasil.

Recebido em agosto de 2002. 


\section{Abstract}

Even though HD is not an AIDS defining disease, the relative risk (RR) for developing HD is definitively higher in at least some of the subgroups of patients with HIV infecction (HIV pos).

O nce it became evident that there was a clinical entity with overall features distinct from $H D$, one sought to establish HD-H IV epidemiological profile; to determine if there was a specific subgroup at higher risk for the development of this new entity or a subgroup in which it did not occur; to define the biological tumor profile; and the chance that these changes to influence the clinical features, the profile of therapeutic responses, and disease free survival.

As H IV pos patients present a more aggressive $H$ odgkin D isease and immunological changes that compromise the therapeutic response seen in the general population, the treatment strategy has shifted, in order to reach complete remission (CR) rates and longer survival time with the use of highly effective antiretroviral schemes, adjustments of old chemotherapy schemes, and new therapeutic protocols.

This article presents and analyses the factors involved in HD-HIV pathogenesis and therapeutics. Key words. Hodgkin's D isease; HIV; EBV; epidemiology; pathology; therapy.

\section{INTRODUÇÃO}

A doença de Hodgkin ( $\mathrm{DH}$ ), até a atualidade, não define o diagnóstico de Síndrome de Imunodeficiência Adquirida (AID S), mas tem achados clínicos e biológicos específicos, quando se apresenta em paciente infectado pelo vírus da imunodeficiência humana (HIV). Essa especificidade leva a um prognóstico diferente do estimado para $\mathrm{DH}$ em indivíduos H IV negativo (H IV neg), fato demonstrado em séries que serão citadas em séries diversas.

Apesar de não ser doença definidora de AIDS, o risco relativo (RR) de $D H$ é definitivamente maior em pelo menos alguns subgrupos de pacientes com infecção pelo HIV (HIV pos).

U ma vez que se evidenciou haver surgido uma entidade clínica com características gerais distintas da $\mathrm{DH}$, procurou-se estabelecer o perfil epidemiológico da DH HIV; determinar se haveria um subgrupo específico de grande risco para o desenvolvimento desta nova entidade, ou algum subgrupo em que não ela ocorresse; definir o perfil biológico tumoral e a possibilidade dessas alterações influenciarem características clínicas, o perfil de resposta terapêutica e a sobrevida livre de doença.

Como os pacientes H IV pos se apresentam com Doença de $\mathrm{H}$ odgkin mais agressiva e alterações imunológicas que comprometem a resposta terapêutica obtida na população geral, a estratégia de tratamento para eles tem mudado, de forma a atingir índices de remissão completa (RC) e sobrevida a longo prazo maiores com o uso de esquemas antiretrovirais altamente eficazes, adaptações de esquemas quimioterápicos antigos e novos protocolos terapêuticos.

0 presente artigo tem como objetivo 0 levantamento e análise dos fatores que se encontram envolvidos na patogênese e terapêutica da DH-HIV.

\section{EPIDEMIOLOGIA}

No início dos anos 80, quando as doenças associadas à Síndrome da Imunodeficiência Adquirida (AIDS), infecciosas ou não, inclusive neoplasias como o Sarcoma de Kaposi (SK) e Linfoma não Hodgkin ( $L N H$ ), começaram a ser detectadas em freqüência crescente, não foi observado aumento semelhante da incidência de $D$ oença de Hodgkin ( $D H)$, sendo relatados somente casos isolados. Além da relativa baixa freqüência de $D H$, a definição de uma relação entre $\mathrm{DH}$ evírus da imunodeficiênciahumana (HIV) era prejudicada pelas confusões diagnósticas com LN H . O utro possível agente a contribuir para esta variação de incidência 
esteve no fato de os pacientes com $\mathrm{LNH}$ e SK serem mais velhos na população geral que na população infectada pelo H IV (HIV pos), sendo inesperado diagnosticar-se essas doenças em adultos jovens, o que suscitou grande interesse na época, enquanto que 0 pico de incidência de $D H$ em relação às faixas etárias não mudou nesses doentes. ${ }^{1,2}$

Análises subseqüentes mostraram um leve aumento de incidência de $\mathrm{DH}$ em homossexuais masculinos. ${ }^{2,3} 0$ registro de câncer de N ova Iorque, em 1985, mostrou um aumento marcante em homens que nunca haviam se casado. D ados do programa SEER (Surveillance, Epidemiology and End Results, também nos Estados U nidos) demonstraram que em São Francisco, onde sabidamente havia maior prevalência de HIV pos entre homens jovens, a incidência de $\mathrm{DH}$ celularidade mista (DH -CM) ajustada para idade aumentou em três períodos distintos (1973-1977, 1978-1982, 1983-1987), e este era o subtipo mais comum por volta dos 50 anos. 0 "Estudo de coorte clínico de São Francisco" de1978-1989, queenvolveu 6.704 doentes, foi analisado de forma comparativa com população semelhante do SEER. Em pacientes HIV pos, o Risco Relativo (RR) de $\mathrm{DH}$ ajustado para idade foi $5(95 \%\{\mathrm{Cl}\}, 2,0$ 10,3). ${ }^{4}$

Em relação aos subgrupos que poderiam estar mais sujeitos a desenvolverem $\mathrm{DH}$, os usuários de drogas intravenosas foram citados em alguns estudos como, por exemplo, na Itália, Espanha, França e em prisões de N ova I orque. ${ }^{5}$ As razões para essa correlação ainda estão sendo debatidas. Embora ainda se levante a hipótese da transmissão de agentes infecciosos em sangue estar envolvida na patogênese da $D H$, não se pode excluir, no entanto, a possibilidade de substâncias ainda não identificadas estarem contaminando as drogas injetadas e exercendo atividade imunomodulatória que contribua para 0 desenvolvimento de $\mathrm{DH}$ nesses pacientes.

Análise de dados do SEER, entre 19761988, em N ova Iorque envolvendo mulheres entre 29 e 40 anos não detectou aumento de incidência de $\mathrm{DH}$ nas HIVpos. O RR para SK e LNH manteve-se aumentado nessa população. Em relação aos hemofílicos, 3.041 pacientes foram analisados de 1978-1989 nos
EU A, e não se detectou aumento da incidência de $\mathrm{DH} .{ }^{4}$ Já um outro estudo publicado encontrou aumento do $R R$, mas sem significado estatística. ${ }^{5}$

A Tabela 1 resume os dados de vários estudos publicados sobre o risco relativo de DH em indivíduos HIV pos.

Tabela 1. Risco relativo de $\mathrm{DH}$ em pessoas com ou em risco de AIDS em estudos de coorte ou análise de registros.

\begin{tabular}{|c|c|c|c|c|}
\hline Autrr & Pat & Tipo de estudo & 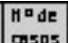 & $m_{0 x}$ \\
\hline Hescol et & En & 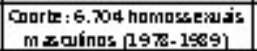 & 8 & $\begin{array}{l}5,0 \\
0,0103\end{array}$ \\
\hline $\begin{array}{l}\text { Reynabs etal } \\
\text { Lueretal }\end{array}$ & EN & 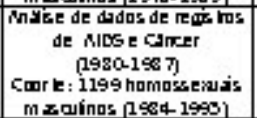 & 16 & $\begin{array}{c}8,8 \\
(5-14,3) \\
19,8 \\
(2,4-715)\end{array}$ \\
\hline Nabin etal & Ex & 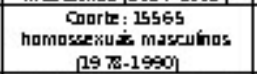 & 18 & $\begin{array}{c}2,5 \\
(1,5-3,9)\end{array}$ \\
\hline & & $\begin{array}{c}\text { coork : Sorounner bres para } \\
\text { HN }\end{array}$ & 3 & $\begin{array}{c}3 \\
(7,8-11,1)\end{array}$ \\
\hline Goeder tetal & 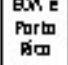 & 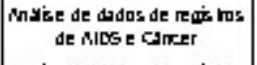 & 140 & $\begin{array}{c}7,6 \\
(4,1-13,1)\end{array}$ \\
\hline $\begin{array}{l}\text { Francexdi etal } \\
\text { Gruich etal }\end{array}$ & 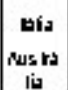 & 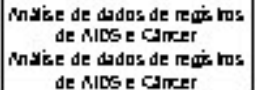 & $\begin{array}{l}11 \\
9\end{array}$ & $\begin{array}{c}2,9 \\
(4,4-16) \\
12,3 \\
4,3,8)\end{array}$ \\
\hline Semaino e tal & $\begin{array}{l}\text { bijae } \\
\text { Fark, }\end{array}$ & 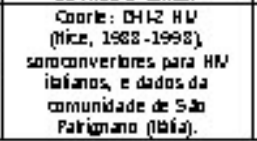 & 7 & $\begin{array}{r}8,7 \\
(2,4-18,0)\end{array}$ \\
\hline
\end{tabular}

\section{PATOLOGIA}

A DH-HIV tem achados patológicos distintos, com distribuição dos subtipos histopatológicos diferentes do global, predominando os subtipos desfavoráveis. ${ }^{1,4}$

A DH -predomínio linfocitário nodular (DH -PLN) e D H clássica (D H C) são as duas categorias da DH, de acordo com a classificação mais recente, da O rganização M undial de Saúde (O M S). ${ }^{7}$ A D HC inclui esclerose nodular (DH -EN), o subtipo rico em linfócitos, a celularidade mista ( $\mathrm{DH}-\mathrm{CM}$ ) e a depleção linfocitária (DH -D L). Já a D H HIV tem como mais freqüentes os subtipos histopatológicos mais agressivos da D HC: $D H-C M, D H-D L$ e casos de DH rica em células de Reed-Sternberg (RS), sendo extremamente incomum o predomínio linfocitário nodular.

$\mathrm{N}$ as avaliações retrospectivas, utilizou-se para descrição, na maior parte dos estudos já liberados, a classificação histopatológica de Rye. Em várias séries, o subtipo mais 
freqüentefoi a D H -CM (41\%-100\%). A D H EN foi bem menos freqüente que em pacientes H IV neg, variando de $0 \%-40 \%$ dos casos. A D H -PL ocorretambém em 0\%-40\%, enquanto a DH-DL é encontrada em $20 \%$ dos casos. ${ }^{1,4,5}$

A célula neoplásica da DH-PLN é denominada linfócito e histiócito $(L \& H)$, enquanto a da DHC se denomina célula de RS. As células $L \& H$ e RS, além de diferentes morfologias e imunofenotipagens, têm padrões de infecção pelo vírus do EpsteinBarr (EBV) distintos. As células $L \& H$ expressam CD 20, CD 45 e antígeno EM A; e as RS expressam CD 15 e CD 30, e são CD 45 negativas. A infecção pelo EBV usualmente só está presente nas células RS, que expressam a proteína LM P-1. N a AIDS, a célula RS é caracterizada por alta freqüência de associação ao EBV $(80 \%-100 \%) .^{5}$

A D H -PLN se diferencia da D H C também pelas células $T$ reativas subjacentes, que, na DH-PLN são CD 57pos, mas CD 40Lneg; e, na DH-CM, CD 40Lpos. No indivíduo HIVpos, a expressão de CD 40L ocorre raramente e, quando ocorre, distribui-se de forma esparsa, não se encontrando rosetas de células CD 40L em torno das células RS.

0 background celular na DHC é bastante diferente entre os indivíduos H IV pos e HIV neg, com inversão da relação de células T CD 4/CD 8 em todos subtipos morfológicos e em todos fenótipos convencionais da doença ${ }^{5}$ e a proliferação de células fibrohistiocitóides estromais que se agrupam em bandas, envolvendo áreas nodulares e mimetizando o padrão clássico de esclerose nodular sem no entanto haver reação de esclerose polarizada. ${ }^{7,8}$

As células RS são mais facilmente encontradas nos pacientes H IV pos. ${ }^{7}$ I sso, associado à rarefação de componentes reativos, causa confusão diagnóstica, principalmente com linfoma anaplásico. A análise imunofenotípica é suficiente para uma correta classificação, sendo o linfoma anaplásico usualmente CD 30pos, CD 45pos e CD 15neg. ${ }^{4,9}$

\section{ACHADOS BIOLÓGICOS E PATOGENIA}

$\mathrm{Na}$ D H -HIV, como descrito anteriormente, há uma alta freqüência de associação ao EBV, variando de $80 \%$ a $100 \%$ dos casos. 0 genoma do EBV em tais casos é relatado como epissomal e clonal. Já nos pacientes HIV neg, a detecção de EBV ocorre em 40\% dos casos nos países do 0 cidente, aumentando em países em desenvolvimento (o que sugere um papel para fatores geográficos ou étnicos na associação do EBV com DH). 0 subtipo mais freqüentemente associado ao EBV é a D H-CM . A distribuição por faixa etária também varia, sendo mais prevalente em crianças e idosos quando comparados a adultos jovens. ${ }^{1,5}$

Estudos mais antigos evidenciaram seqüências de $D N A$ de EBV em virtualmente todos os casos de DH-HIV, oferecendo evidência direta da relação causal deste vírus na patogênese da DH. Esses estudos foram posteriormente confirmados por técnicas baseadas na detecção de pequenos transcritos virais e RN A nucleares codificados pelo EBV (EBER) que estão em grandenúmero decópias presentes em células infectadas latentes. A análise por hibridização - Southern blot demonstrou epissomos de EBV clonais em pacientes HIV pos. Este achado é consistente com a expansão monoclonal em células carreadoras de EBV na maioria dos casos de DH -HIV e indica que a infecção pelo EBV ocorreu antes da expansão clonal das células RS. Também se demonstrou que um mesmo clone celular infectado pelo EBV com achados morfológicos de DH -célula RS persistia em várias localizações não contíguas desta doença. Todos esses achados colocaram o EBV como fator relevante para a patogênese daD H -HIV. ${ }^{5}$

$\mathrm{N}$ os indivíduos imunocompetentes, 0 vírus expresso por células RS parece estar estritamente latente, com o é demonstrado pela expressão de EBER e ausência de antígenos líticos, como o gp350/220, VCA e EA. Por outro lado, a expressão do transativador BZLF1 foi demonstrada em poucos casos de DH na célula RS, indicando que, quando o ciclo replicativo ou ativação ocorre, geralmente leva ao abortamento da produção viral. Evidências sugerem que imunodefi- 
ciência grave poderia permitir que 0 vírus saísse do estado de latência para o de ativação, como no LNH-EBV em imunodeficientes, nos quais há replicação viral em $60 \%$ dos casos. $\mathrm{N}$ a DH -H IV isso não ocorre, estando o EBV estritamente latente. Essa discrepância pode estar nas gradações do estado imunológico do paciente, tipo e diferenciação celular das células infectadas pelo EBV ou propriedades específicas virais. ${ }^{5}$

$O$ padrão delatência do EBV em diferentes doenças varia amplamente. $\mathrm{Na} \mathrm{DH}$ há expressão de EBER, antígeno nuclear do EBV (EBN A)-1, LM P-1 eLM P-2, mas não EBN A-

2. Essa forma de latência viral é denominada latência II. U m padrão mais restrito se encontra no linfoma de Burkitt (latência I), em que somente EBER e EBNA-I são expressos. No entanto, células imortalizadas EBV poslinfoblastóides delinhagem B eLN H em imunodeficientes usualmente expressam todos os genes latentes codificados pelo EBV (padrão de latência III). C omo na população geral de DH, a DH-HIV também tem um padrão de expressão latência II, o que pode se explicar por diferentes graus de imunossupressão, visto que normalmente a DH -HIV se manifesta em fases mais iniciais do que o LNH-HIV, com níveis de CD 4 superiores e sem doenças compatíveis com AIDS prévias.

A expressão de EBER e EBN A-1 ocorre em todas as células com infecção latente pelo EBVm inclusive linfócitos normais carreadores de EBV. Já a expressão de LM P. 1 não ocorre de forma estável em condições fisiológicas. Estudos experimentais identificaram a LM P-1 como um oncogene viral. Análise genética de EBV recombinante demonstrou que a LMP-1 é essencial para imortalização de linfóticos B primários. N essas células, a LM P-1 pode induzir a várias alterações fenotípicas associadas à infecção pelo EBV, incluindo o aumento da adesão homotípica, a upregulation de moléculas de adesão de antígeno de função leucocitária (LFA)-1 e de moléculas de adesão intercelular (IC AM )-1, (LFA-3), expressão de marcadores de ativação de células $B(C D$ 30, CD 23, CD 40 e CD 71), e de genes antiapoptóticos ( $\mathrm{BCl}-2$, $B c l x L, M c 11, A 20)$. Além da adição de marcadores fenotípicos associados a D H - células RS, a expressão de LM P-1 pode estar associada às alterações citomorfológicas dessas células. A LM P-1 interage com proténas do citoesqueleto como a vimentina, e também age como mediadora da downregulation do CD 99, o que induz à produção de células com fenótipo DH-RS. ${ }^{5}$

$O$ utro achado importante relacionado ao LM P-1 foi a deleção de 30-bp na porção final 3' deste gene ser detectada em 10\%-40\% dos casos de DH em pacientes HIVneg. Os pacientes com essa deleção tinham um padrão de comportamento da DH mais agressivo. A $\mathrm{DH}-\mathrm{H}$ IV tem maior prevalência dessa deleção, o que levanta a possibilidade de ela estar contribuindo para o comportamento mais agressivo da DH nos indivíduos HIV pos.

Existem dois subtipos distintos de EBV, denominados A e B (ou 1 e 2). 0 tipo A é mais freqüente na população ocidental e 0 tipo $B$, na África equatorial. Além das variações geográficas, o tipo $B$ é encontrado em swabs de orofaringe de indivíduos saudáveis e em sangue periférico de indivíduos infectados pelo H IV. $\mathrm{N}$ a avaliação de DH associada ao EBV, encontrou-se uma grande predominância do tipo $\mathrm{A}$ (em $56 \%$ $100 \%$ dos casos). Em contraste, $50 \%$ dos pacientes com DH-H IV carregam o tipo B, achado semelhante ao encontrado para 0 LN H -H IV, indicando que, no contexto da imunodeficiência pelo HIV, tanto o tipo 1 como o 2 do EBV são capazes de contribuir para o desenvolvimento da $\mathrm{DH}$.

Em relação à origem da células $\mathrm{RS}$ na $\mathrm{DH}$, vários estudos foram feitos com base em marcadores biológicos para a identificação de diferentes estágios de maturação do linfócito B. Foram analisados linfonodos de pacientes com D H -H IV pose comparados aos com D H HIVneg, quanto ao padrão de expressão de BCL-6 (fator de transcrição expresso em células $B$ do centro germinativo - CG) e CD 138/syndecan-1(syn-1), um proteoglican associado com a diferenciação terminal, pósCG. As células $L \& H$ da DH-PLN estavam associadas ao fenótipo BCL6pos/syn-1neg. Já as células RS raramente expressavam 0 fenótipo BCL6pos/syn-1neg, o que ocorria somente em alguns pacientes H IV neg com DHC. Este fenótipo não foi encontrado 
naqueles com DH-HIV. O fenótipo mais prevalentetanto nos indivíduos H IV pos como nos H IV neg com D H C foi o BCL6-/syn-1pos.

$O$ background celular reativo em células RS BC L6neg/syn-1pos na D H não associada ao HIV também é composto por células T que expressam CD 40L (a interação CD 40/ CD 40L poderia modular a diferenciação do clone neoplásico e promover a downregulation do BCL-6 em células RS). $N$ os indivíduos HIV pos, as células RS expressam CD 40, mas não CD 40L. Porém, há evidências de que, in vitro, a LM P-1 também poderia modular a downregulation do BCL-6 em células com fenótipo de CG e, virtualmente, a LM P está presenteem todos os casos de D H -H IV, sendo funcionalmente homóloga ao CD 40,5,9

N ovos marcadores histogênicos vêm sendo avaliados recentemente: 0 M U M 1/IRF4 (mieloma múltiplo $1 /$ fator regulador de interferon 4) tem sido acrescentado ao painel de marcação imunofenotípico de linhagem $B$. Comparação topográfica do BCL 6 e M U M 1 no $C G$ revela que o BCL6 é expresso desde a entrada das células neste centro até sua saída deste. O MUM 1 inicia sua expressão nos centrócitos e permanece durante a maturação pós-CG. Todos os centroblastos e a maioria dos centrócitos expressam BCL6pos/ M U M 1neg/syn-1neg (pequena parte das células do CG na zona clara, e morfologicamente identificadas como parcela dos centrócitos, expressam BCL6neg/M U M 1pos/syn-1neg). Após a saída do $C G$, as células mantêm a expressão de MUM-1 e passam a expressar syn-1. A DH-HIV tem fenótipo BCL-6/M U M -1neg-pos/syn-1pos, corroborando o conceito de refletirem células B pós-CG.

\section{CARACTERÍSTICAS CLIINICAS}

As características mais marcantes da D H H IV são a agressividade e a extensão da doença ao diagnóstico. $\mathrm{N}$ o indivíduo H IV neg, a doença se espalha tipicamente para órgãos nodais contíguos. N os pacientes HIV pos, 0 acometimento não contíguo éfreqüentemente observado (massa pulmonar sem linfoadenomegalia mediastinal; acometimento hepático com baço livre de doença; etc). Sintomas B ocorrem em 70\%-96\% dos pacientes (febre, sudorese noturna ou perda de mais de $10 \%$ do peso corporal normal). Estágios III e IV de Ann Arbor são vistos em $74 \%-92 \%$ dos casos, com $60 \%$ dos pacientes apresentando doença extranodal como apresentação inicial. A medula óssea (M O) está acometida em 40\%-50\% das biópsias. 0 acometimento hepático ou esplênico é identificado, respectivamente, em 15\%-40\% e 20\% dos pacientes. ${ }^{1,5}$ Localizações incomuns de acometimento primário são descritos, como o sistema nervoso central - SN C, pele, pulmão, reto e língua. 0 acometimento mediastinal é raro e se observa em 13\%-23\% dos casos HIVpos e em $58 \%-71 \%$ dos HIV neg.

A DH se desenvolve mais precocemente no contexto da infecção pelo H IV, quando comparada ao Linfoma D ifuso de Grandes Células (LDGC), quando se consideram os níveis de CD4. As séries de DH-HIV descrevem a contagem média de CD 4 variando de $275 / \mathrm{mcl}$ a $306 / \mathrm{mcl}$.,10

N o momento da apresentação inicial, a maioria dos pacientes com DH $165 \%$ em algumas séries) tem linfoadenomegalia generalizada persistente (LGP) e, em $50 \%$ dos casos, encontra-se tanto DH quanto LGP no mesmo grupo nodal. Então, o aumento de tamanho de um órgão nodal em um paciente com LGP deve ser investigado exaustivamente, em busca de outra(s) linfoadenomegalia(s), acometimento hepático ou de medula óssea - que devem ser biopsiados para definição diagnóstica. Em grande parte dos casos, são necessárias múltiplas biópsias antes de se chegar a um diagnóstico definitivo. 0 utro ponto relevamte é ter-se atenção ao fato de que pacientes com DH -HIV, quando avaliados por método de imagem, podem ter LGP, em retroperitônio por exemplo, erroneamente diagnosticada como DH. A LGP, no entanto, raramente é encontrada em região mediastinal ou hilar, diminuindo enormemente a chance de erro nessas topografias.

Andrieu et al ${ }^{11}$ compararam 45 casos de D H coletados pelo registro francês entre 1987 e 1989 com coorte de407 indivíduos H IV neg e com DH. Diferença significativa foi encontrada em relação às seguintes variáveis: 
estágios clínicos avançados (75\%/33\%), prevalência de CM (49\%/20\%) e ausência de doença mediastinal $(87 \% / 29 \%)$.

Tirelli et al ${ }^{1}$ descreveram 114 pacientes de um total de 39 centros com DH -HIV pos e os comparou a 104 pacientes controles $\mathrm{D} \mathrm{H}$ HIV neg. A mediana de idade do primeiro grupo foi de 29 anos e $90 \%$ deste grupo de doentes eram do sexo masculino, sendo ele mais jovem que o segundo, e com maior prevalência de estágio IV, doença extranodal e sintomas constitucionais.

As infecções oportunistas, incomuns em pacientes HIV neg, ocorrem com alta freqüência nos HIV pos: Pneumonia por Pneumocystis carinii (PCP), micobacterioses, infecção pelo citomegalovírus - CMV, toxoplasmose, candidíase disseminada, criptosporidiose, criptococose e outras doenças são a causa de óbito em 35\% dos pacientes. ${ }^{4,11}$

As séries italianas foram as maiores publicadas até o momento. Entre novembro de 1986 esetembro de 2000 , foram coletados dados de 206 pacientes do GICAT (Grupo italiano cooperativo em AIDS e Tumores) com DH H IV. Destes pacientes, 180 eram homens (87\%), com mediana de idade de 31 anos (variando de 19-63 anos). Em relação ao grupo de risco, a maioria dos pacientes era usuária de drogas intravenosas, o que está de acordo com a epidemiologia da infecção pelo H IV na Itália. $\mathrm{N}$ a ocasião do diagnóstico de D H , 74 em 188 (39\%) eram assintomáticos para o HIV e 44 em 188 pacientes $(23 \%)$ tinham diagnóstico de AIDS, 33 em 188 (18\%) de LGP, e 37 em 188 (20\%) de complexo relacionado a AIDS. A mediana de CD 4 ao diagnóstico foi de 231/ mcl (variação de $4-1 \cdot 100 / \mathrm{mcl}$ ). As demais características desses pacientes encontram-se relacionadas na Tabela $2 .{ }^{10}$
Tabela 2. Achados clínicos de 206 pacientes com DH-HIV avaliados pelo GICAT.

\begin{tabular}{|c|c|}
\hline Subtipo de $\mathrm{DH}$ & Freqüüencia (\%) \\
\hline $\bar{F}$ & $4(2)$ \\
\hline rE & $48(27)$ \\
\hline$M$ & 96 (53) \\
\hline ம & $32(18)$ \\
\hline Estigio (Axn $A$ (tox) & \\
\hline 1 & 9 (5) \\
\hline II & 27 (14) \\
\hline III & $56(28)$ \\
\hline $\mathrm{N}$ & $108(54)$ \\
\hline Enwokiments extranodal & $130(75)$ \\
\hline Medula 6 ssea & $78(39)$ \\
\hline $\mathrm{Eag}$ & 62 (31) \\
\hline Figado & $37(19)$ \\
\hline Pulmáo & $11(6)$ \\
\hline Ossog & 7 (4) \\
\hline Tratu gastrintestinal & $3(2)$ \\
\hline Pele & 2 (1) \\
\hline STE & 2 (1) \\
\hline Anel de Wableyer & $1(0.5)$ \\
\hline Testialos & $1(0.5)$ \\
\hline Sintomas E (n = 188) & $150(80)$ \\
\hline
\end{tabular}

Fonte: Referência ${ }^{10}$

Em adição aos prognósticos classicamente usados para D H , como doença bulky, invasão da medula óssea, acometimento inguinal, desidrogenase láctica (DHL) sérica aumentada, idade do doente maior que 40 anos, velocidade de hemossedimentação (VHS) elevada, anemia, nível de albuminemia e estágio de Ann Arbor, devem ser incorporados para a estimativa da sobrevida dos doentes com D H - H IV pos a contagem de células CD 4 ao diagnóstico e a existência de doença compatível com AIDS prévia ao diagnóstico de D H -HIV. A série antes citada, conduzida por Tirelli et al, identificou esses como marcadores de pior prognóstico para a DH -H IV. N essa série, pacientes com CD 4 menor que $250 / \mathrm{mcl}$ tiveram sobrevida mediana de 11 meses e os com CD 4 maior que $250 / \mathrm{mcl}$, de 38 meses $(p<0,002)$. $N$ os indivíduos com AIDS prévia, a mediana de sobrevida foi de sete meses; e, nos sem doença compatível com AIDS ao início do tratamento, de 20 meses $(p<0,001){ }^{4}$

\section{TRATAMENTO}

Atualmente, a maioria dos pacientes com D H pode ser curada. M esmo pacientes em estágio IV de Ann Arbor e com o índice prognóstico 5 usado por $\mathrm{H}$ asenclever eD iehl ${ }^{12}$ alcançam mais de $42 \%$ de sobrevida livre de doença. A quimioterapia com ABVD 
(adriamicina/doxorrubicina, bleomicina, vimblastina e dacarbazina) associa-se a $70 \%$ $80 \%$ de RC e a $60 \%-70 \%$ de sobrevida a longo prazo, em caso de pacientes H IV neg e com DH avançada. $\mathrm{N}$ o entanto, pacientescom DH-HIV evoluem de forma menos satisfatória. ${ }^{10} \mathrm{~A}$ maioria dos pacientes com D H -H IV se apresenta com doença avançada, sendo tratada com quimioterapia. As complicações são várias. A imunodeficiência associada ao HIV aumenta as taxa de infecções oportunistas durante a quimioterapia e também após a mesma, visto não poder se esperar a recomposição imediata do sistema imunológico. As citopenias ligadas ao HIV, principalmente a leucopenia pela mielodisplasia (MDS) ou devido ao uso de análogo de nucleosídeos (antiretrovirais), dificulta 0 uso de doses ideais de agentes quimioterápicos.

Estudos retrospectivos sobre D H - H IV demonstraram uma menor taxa de RC, baixa tolerância à quimioterapia e necesAID Sde de se reduzir doses ou atrasar os ciclos de quimioterapia. ${ }^{9}$ O GICAT analisou 41 pacientes tratados com MOPP ou M OPP/ ABVD e encontrou maior taxa de RC $(65 \%$ versus $46 \%$ ) no segundo grupo. Além disso, observou-se menor taxa de complicações infecciosas neste grupo. Também pelo GICAT, ${ }^{13}$ foi realizado estudo prospectivo com 17 pacientes recém diagnosticados com DH -HIV, entre março de 1989 e março de 1992, tratados com EVB (epirrubicina- 70mg/ $\mathrm{m}^{2}$, vimblastina - $6 \mathrm{mg} / \mathrm{m}^{2}$ e bleomicina - 10 $\mathrm{U} / \mathrm{m}^{2}$, em $1 \mathrm{dia}$ ) repetidos por seis ciclos com intervalo mínimo de 21 dias. 0 s pacientes foram divididos em dois grupos. 0 primeiro grupo, sem doenças oportunistas prévias e Performancestatus(PS) pelo ECO G menor que 3 (Grupo A); e o segundo grupo, com PS igual ou maior que 3 ou com doença oportunista prévia (G rupo B) que receberia $50 \%$ da dose de epirrubicina e de vimblastina e teria a zidovudina (AZT) introduzida no início da quimioterapia. 0 grupo $A$ receberia $A Z T$ somente após o 30 ciclo de quimioterapia. $\mathrm{Na}$ análise dos dados, atingiu-se RC em $67 \%$ dos pacientes do grupo $\mathrm{A}$ e em $20 \%$ do grupo B. A mediana de sobrevida para todo grupo foi de 11 meses, sendo que $55 \%$ dos pacientes estavam vivos dois anos após o início da quimioterapia.

No intuito de melhorar os resultados obtidos pelo GICAT, em janeiro de 1993 o Intergrupo Europeu deD H -H IV iniciou outro ensaio clínico usando como esquema quimioterápico EVB e adicionando prednisona $40 \mathrm{mg} / \mathrm{m}^{2}$, por 5 dias. Foi associada AZT (250mg de $12 / 12 \mathrm{~h}$ ) ou didanosina - DDI (200mg ou 300mg de 12/ $12 \mathrm{~h})$ desde o começo dos ciclos de quimioterapia. 0 fator estimulante do crescimento de colônias de granulócitos - G CSF (5mcg/ $/ \mathrm{Kg} / \mathrm{dia})$ foi usado do dia 6 ao 20, em todos os ciclos e o tratamento profilático de PC $P$ também foi acrescentado. Até agosto de 1997, 35 pacientes foram consecutivamente alocados. A mediana do PS foi de 1 (extremos de 1 a3), 26\% dos pacientes tinham diagnóstico de AIDS prévio. 0 percentual de resposta à quimioterapia foi de $91 \%$, sendo $74 \%$ de RC e $17 \%$ de resposta parcial (RP). Infecção oportunista ocorreu em $8 \%$ dos pacientes durante ou após a quimioterapia, em 22 meses de acompanhamento. N eutropenia grau III ou IV foi encontrada em $32 \%$ dos pacientes, e trombocitopenia em $10 \%$ deles. $\mathrm{D}$ os pacientes que atingiram RC, $38 \%$ recaíram. DH isolada ou em associação a infecção oportunista foi causa de óbito em $48 \%$ e $9 \%$ dos pacientes, respectivamente. $A$ sobrevida mediana foi de 16 meses; a taxa de sobrevida, de $32 \%$; e a sobrevida livre de doença, de $53 \%$ em 36 meses. ${ }^{4,10}$

U m estudo prospectivo multicêntrico, não randomizado, conduzido de maio de 1992 a agosto de 1996, foi publicado por Levine et al ${ }^{14}$ e que incluiu 21 pacientes DH-HIV tratados com esquema ABVD padrão e resgate com G-CSF. N este estudo, nenhum pacienteteveuso deH ighly activeantiretroviral therapy (HAART) associado (início do tratamento pré-advento de inibidor de protease). As características dos pacientes acompanham a descrição da literatura, com doença agressiva à apresentação inicial: Estágio IV em $67 \%$, sintomas constitucionais em $90 \%$ e medula óssea invadida em $57 \%$ dos doentes. A contagem média de células CD 4pos foi de $113 / \mathrm{mm}^{3}$ e $29 \%$ dos pacientes tiveram uma doença compatível com AIDS antes do diagnóstico de $\mathrm{DH}$. $\mathrm{Na}$ análise do resultado terapêutico, a taxa de $\mathrm{RC}$ foi de 
$43 \%(90 \% \mathrm{Cl}, 24 \%-63 \%)$ e de $19 \%$ de resposta parcial, ficando em $62 \%$ a taxa total de resposta. A sobrevida média foi de 1,5 anos. Apesar do uso de G-CSF, dez pacientes $(47,6 \%)$ apresentaram neutropenia menor que $500 \mathrm{cel} / \mathrm{mm}^{3}$. No total, nove episódios de infecções oportunistas ocorreram durante a quimioterapia ou logo após o término dela.

O esquema Stanford V (mecloretamina, doxorrubicina, vimblastina, vincristina, bleomicina, etoposido, prednisona e G-C SF) foi estudado em pacientes H IV neg pelo grupo de Stanford, Califórnia, como alternativa para 0 esquema ABVD. ${ }^{15} \mathrm{Em}$ um grupo de 65 pacientes, obteve-se taxa de sobrevida livre de doença de $87 \%$ com esse esquema, e a mediana da sobrevida em três anos foi de $96 \%$. Spina et al ${ }^{16}$ apresentaram achados referentes a 50 pacientes HIV pos com diagnóstico recente de $\mathrm{DH}$ e tratados com 0 esquema Stanford V. As características dos pacientes no início do tratamento foram: mediana de idade de 36 anos com extremos de 28 e 63 anos, sendo que $46 \%$ dos pacientes eram usuários de drogas, $20 \%$ tinham doença compatível com AIDS prévia, mediana de CD 4pos de $235 / \mathrm{mm}^{3}$ (extremos de 32-1.008), mediana de HIV-RN A plasmático (carga viral) de 5.841 cópias $/ \mathrm{ml}$. Vinte eoito por cento dos casos estavam em estágio II e 46\%, em estágio IV, sendo que $42 \%$ dos casos tinham invasão de medula óssea e o índice prognóstico internacional foi de 0 em 2\%, 1 em 18\%, 2 em 26\%, 3 em 24\%, 4 em 8\%, 5 em $20 \%$ e 6 em $2 \%$. Os pacientes que atingiram RC ou RP receberam radioterapia com campos envolvidos, em caso de doença mediastinal grosseira (bulky disease), ou qualquer massa maior que $5 \mathrm{~cm}$ de diâmetro, ou se doença esplênica presente. 0 esquema antiretroviral tríplice foi administrado a todos pacientes, junto com o tratamento profilático da PCP e candidíase. 0 G-CSF foi usado na dose de $5 \mathrm{mcg} / \mathrm{Kg} /$ dia nos dias 3-13 e 17-26 de cada ciclo. Sessenta e oito por cento dos pacientes completaram o tratamento sem doença progressiva, em $10 \%$ dos casos. D os 39 em RC, 18\% recaíram em intervalo de 12 meses (com limites de 1-46 meses). $\mathrm{Na}$ ocasião da publicação inicial do estudo, 72\% dos pacientes estavam vivos e $68 \%$ livres de doença em dois anos. A D H foi causa deóbito em 10 dos 14 pacientes que faleceram. N eutropenia grau III ou IV ocorreu em $82 \%$ dos pacientes; PCP ou micobacteriose, em $8 \%$; mucosite, em $42 \%$ e neuropatia periférica, em $44 \%$.

Os dados inicialmente sugerem que Stanford $V$ seria mais efetivo que o ABVD padrão no tratamento da $\mathrm{DH}-\mathrm{H} \mathrm{IV}$, porém os dois estudos têm características populacionais e de condutas muito diferentes, por exemplo: mediana de CD 4 do primeiro grupo de 235 células $/ \mathrm{mm}^{3}$ e, do segundo, de 113 células/ $\mathrm{mm}^{3}$; uso de HAART associado, no primeiro grupo, mas não no segundo. Além disto, apesar da taxa de RC ser maior no grupo tratado com Stanford V (78\% versus 43\%), a mediana de sobrevida dos dois grupos não variou tanto: 18 meses no ABVD e 24 meses no outro grupo. ${ }^{14,16} \quad 0$ esquema Stanford $V$ está sendo avaliado atualmente no Consórcio de AID S e malignidade.

Tem sido investigado 0 uso de quimioterapia em altas doses com resgate de células progenitoras de medula óssea para pacientes HIV pos com linfoma. Até o momento, não há tratamento de $2^{\mathrm{a}}$ linha ou de resgate estabelecido para pacientes cuja quimioterapia inicial falhou. Gabarre et al ${ }^{17}$ apresentaram resultados de 11 pacientes tratados entre novembro de 1994 e outubro de 2000. O ESH AP (etoposido, cisplatina, citarabina e metilprednisolona) foi usado como quimioterapia de segunda linha e a mobilização com G-C SF foi feita em 10 pacientes, e coleta de medula óssea em 1 doente. BEAM (BCNU, etoposido, arabinosídio e melfalan) foi o esquema de condicionamento utilizado para cinco pacientes, enquanto seis o receberam associado à irradiação corporal total.

A composição deste grupo variava da seguinte forma: $D H$ recaída, 5 casos; Linfoma de Burkitt - LB, 3 casos (um em RP, um em recaída de doença, e um com doença resistente); Linfoma Imunoblástico - LI, 2 casos (um em recaída e um em RP); e 1 paciente em recaída de Linfoma $D$ ifuso de Grandes Células - LDGC. 0 esquema H AART foi administrado em 10 pacientes. A pega da célula tronco foi adequada em todos eles. A mediana de células CD 34pos a serem recebidas foi de $5,8 \times 10^{6}$ células $/ \mathrm{kg}$. As 
medianas de tempo para a obtenção de granulócitos acima de $500 / \mathrm{mm}^{3}$ e plaquetas acima de $20.000 / \mathrm{mm}^{3}$ foram, respectivamente, de 12 e 11 dias. $\mathrm{N}$ a avaliação póstransplante, sete pacientes estavam em RC (4/ 5 dos casos de $D H, 1 / 3$ dos de $L B, 1 / 2$ dos de $\mathrm{LI}$ e o de LDCG). Todos os pacientes com doença refratária morreram pelo linfoma 1 a 6 meses após o transplante. Dos sete pacientes que atingiram $\mathrm{RC}$, o único paciente tratado antes do HAART faleceu de AIDS. No grupo tratado com HAART, um faleceu por AID S; um recaiu do linfoma aos 9 meses pós-transplante associado a anemia hemolítica grave; e quatro pacientes (36\%) estavam vivos e sem evidência de linfoma aos 5, 10, 20 e 28 meses pós-transplante autólogo de células progenitoras. A sobrevida mediana de todo 0 grupo foi de 7 meses. $\mathrm{N}$ enhuma infecção oportunista grave ocorreu, apesar de dois pacientes apresentarem reativação do CM V. O s autores concluem que a inclusão de inibidores de protease e 0 uso de esquema HAART no tratamento de pacientes com linfoma e HIV possibilitaram melhora imunológica e aumento de linfócitos CD 4, reduzindo a chance de infecções oportunistas. A inclusão de fatores de crescimento hematopoéticos pode permitir o uso de quimioterapia mais potentes, compatíveis com o grau de agressividade do linfoma nestes pacientes, o que possivelmente levará a mai ores taxas de RC e aumento da sobrevida livre de doença.

\section{CONCLUSÃO}

Vários estudos publicados demonstram que pacientes com HIVpos têm maior RR para 0 desenvolvimento de DH. As particularidades dessa relação vêm sendo levantadas. $\mathrm{N}$ ão se pode, até 0 momento, concluir sobre a extensão do envolvimento do EBV e LMP-1 na gênese e no perfil de agressividade da DH-HIV, mas seu papel parece estar definido por sua alta prevalência nessa população. As manifestações clínicas de DH podem ser bastante distintas nos H IV neg e HIV pos, o que é um alerta para a suspeita diagnóstica.

A melhora imunológica que se conseguiu para esses pacientes com o uso do esquema
HAART e a conseqüente elevação do nível de CD 4 (mesmo sem a normalização da carga viral) diminuiu o número de infecções oportunistas, possivelmente permitindo o uso de quimioterapias mais potentes que as oferecidas no inicialmente, com o aumento na expectativa de vida. Protocolos estão sendo conduzidos, inclusive com quimioterapia em altas doses e resgate de células progenitoras ou mesmo transplante alogênico de medula óssea. O limiar para a agressividade da quimioterapia a ser oferecida, assim como 0 suporte profilático às infecções, ainda não está estimado para esses pacientes.

Novos estudos prospectivos são necessários para se otimizar o tratamento da DH-HIV.

\section{REFERÊNCIAS BIBLIOGRÁFICAS}

1. Tirelli U, ErranteD, D olcetti R, etal. H odgkin's disease and human immunodeficiency virus infection: clinicopathologic and virologic features of 114 patientsfrom the Italian C ooperative Group on AID S and tumors. J Clin O ncol 1995;13:1758-67.

2. Prior F, Goldberg AF, Conjalka MS, et al. $H$ odgkin's disease in homosexual men. An AID S-related phenomenon? Am J M ed 1986;81(6):1085-8.

3. Serraino $D$, Boschini $A, C$ arrieri $P$, et al. C ancer risk among men with, or at risk of, H IV infection in southern Europe. AIDS 2000;14(5):553-9.

4. Spina M , Vaccher E, Nasti G, Tirelli U. Human immunodeficiency virus associated H odgkin's disease. Eur J Cancer 2001; 37(10):1276-87.

5. Dolcetti R, Boiocchi M , Gloghini A, C arbone A. Pathogenic and histogenic features of H IVassociated H odgkin's disease. Eur J Cancer 2001;37(10):1276-87.

6. JaffeE, H arrisN L, D iebold J, et al. World H ealth $O$ rganization Classification of neoplasic diseases of the hematopoietic and lymphoid tissues: a progress report. Am J Clin Pathol 1999; 111(Suppl 1):S8-12.

7. ReeHJ, Strauchen JA, Khan AA, et al. H uman immunodeficiency virus associated $H$ odgkin's disease. Clinicopathologic study of 24 casesand preponderance of mixed cellularity typecharacterized by theoccurrence of fibrohistiocytoid 
stromal cells. C ancer 1991;67:1614-21.

8. Bellas C, Santón A, M anzanal A, et al. Pathological, immunological and molecular features of H odgkin's disease associated with H IV infection. Comparison with ordinary H odgkin's disease. Am J Surg Pathol 1996;20:1520-4.

9. Tirelli U, Vaccher $E$, Zagonel V, et al. C 30 (K i1) positive anaplasic large cell lymphomas in 13 patients with and 27 patients without human immunodeficiency virus infection. The first comparative clinicopathologic study from a single institution that also includes 80 patients with othersimmunodeficiency virus-related systemic lymphomas. J Clin O ncol 1995;13:373-80.

10. Vaccher E, Spina M , Tirelli U. Clinical aspects and manegement of H odgkin's disease and other tumours in HIV- infected individuals. Eur J Cancer 2001;37(10):1306-15.

11. Audrieu JM , Roithmann S, Tourani JM , et al. H odgkin's disease during H IV-1 infection: the French Registry experience. French Registry of HIV- associated tumors. Ann O ncol 1993;4:635-41.

12. H asenclever D, D iehl V. A prognostic scorefor advanced H odgkin's disease. International prognostic factors project on advanced Hodgkin's disease. N Engl J Med 1998;339(21):1506-16.

13. Errante D, Tirelli U, G astaldi R, et al. Combined antineoplasic and antiretroviral therapy for patients with $\mathrm{H}$ odgkin's disease and human immunedefiency virus infection: a prospective study of 17 patients. Cancer 1994;73:437-44.

14. Levine Am, Li P, Cheung T, et al. Chemotherapy consisting of doxorrubicin, bleomycin, vinblastine, and dacarbazine with granulocyte colony stimulating factor in H IV infected patients with newly diagnosed $\mathrm{H}$ odgkin's: a prospective, multi-institutional AIDS. C linical Trials Group Study (ACT G 149). J Acquir Immune D efic Syndr 2000;24:444-50.

15. H orning S, Rosenberg AS, H oppe RT. Brief chemotherapy (Stanford V) and adjuvant radiotherapy for bulky or advanced H odgkin's disease: an update. Ann O ncol 1996;7 Suppl 4:105.

16. Spina M , G abarreE, Vaccher E, et al. Feasibility of the integration of Stanford $V$ chemotherapy regimen with highly active antiretroviral therapy (H AART) and G - CSF in patients with HD and H IV infection [abstract 24]. Program and abstracts of the5th I nterna tional M alignancy Conference; 2001 A pr 2325; Bethesda, M aryland.

17. Gabarre J, Azar N , Kotb R, et al. H igh dose chemotherapy(H DC) and autologousstem-cell transplantation (ASCT) for H IV associated lymphoma(Ly) [abstract 23]. Program and abstracts of the 5th International M alignancy $\mathrm{C}$ onference; 2001 Apr 23-25; Bethesda, M aryland. 